\title{
The nature of working memory gating in Parkinson's disease: A multi-domain signal detection examination
}

\author{
Mitchell G. Uitvlugt ${ }^{1}$ - Timothy J. Pleskac ${ }^{2} \cdot$ Susan M. Ravizza $^{1}$
}

Published online: 30 October 2015

(C) Psychonomic Society, Inc. 2015

\begin{abstract}
Distractions are ubiquitous; our brains are inundated with task-irrelevant information. Thus, to remember successfully, one must actively maintain relevant information and prevent distraction from entering working memory. Researchers suggest the basal ganglia-prefrontal pathways are vital to this process by acting as a working memory gate. Using Parkinson's disease as a model of frontostriatal functioning and with signal detection analyses, the present study aims to better characterize the contribution of frontostriatal pathways of this gating process and to determine how it operates across multiple domains. To achieve this, Parkinson's disease patients and healthy controls completed verbal and spatial working memory tasks consisting of three conditions: low-load without distraction; low-load with distraction; and high-load without distraction. Patients were tested both ON and OFF dopaminergic medication, allowing for assessment of the contribution of dorsal and ventral frontostriatal pathways. The results demonstrate that when medication is withheld, Parkinson's patients have a response bias to answer "NO" across all conditions and domains, supporting our hypothesis that the basal ganglia-prefrontal pathways allow or prevent updates of working memory. Contrastingly, medication status affects $d^{\prime}$ in the distraction condition but not in the high- or low-load conditions. We attribute this to stimulus valuation processes that were impaired by dopaminergic medication overdosing the ventral pathway. These findings are both consistent with the hypothesis that the
\end{abstract}

Susan M. Ravizza

ravizzas@msu.edu

1 Department of Psychology, Michigan State University, 316 Physics Road, Room 285C, East Lansing, MI 48824, USA

2 Center for Adaptive Rationality, Max Planck Institute for Human Development, Berlin, Germany working memory gate filters spatial and verbal information before it enters into the working memory system, adding support for the gate being a domain-general mechanism of the central executive.

Keywords Working memory · Gating · Parkinson's disease . Basal ganglia $\cdot$ Distraction

Working memory (WM) requires dynamic control in order to disregard irrelevant information while insuring attendance to task-relevant information. This process is thought to be implemented by a WM "gate." In theory, the gate regulates the influence of incoming stimuli on the WM system, determining if information is allowed entrance or if prior contents should instead be maintained (Miller \& Cohen, 2001). The neural mechanics and mechanisms of a WM gate are, however, not fully understood. Therefore, the present study seeks two aims: to assess the proposed neural mechanisms by which such a gate operates and to determine if it does so similarly across domains - here, verbal and spatial.

The prefrontal cortex (PFC) and the basal ganglia (BG) are argued to provide this gating mechanism (O'Reilly \& Frank, 2006). The direct D1 "Go" and indirect D2 "NoGo" pathways of the BG are believed to implement goal states that are represented in the PFC. Disinhibition of the thalamocortical loops in the "Go" pathway signals a WM update. Without striatal firing, the gate remains closed as now the "NoGo" pathway dominates (Frank, Loughry, \& O’Reilly, 2001). Recent functional magnetic resonance imaging (fMRI) studies support these arguments; that is, activity in the BG is observed when task-relevant items are initially entered into WM at encoding (Chein \& Fiez, 2010; Chen \& Desmond, 2005; Moore, Li, Tyner, Hu, \& Crossom, 2013; Peters et al., 2009) or must be subsequently updated (Fallon \& Cools, 2014; Nee \& Brown, 
2013). Moreover, activity in the PFC and BG was higher during preparation when distraction was expected to occur, perhaps reflecting control processes necessary to encode only task-relevant information (McNab \& Klingberg, 2008).

Studies of distraction effects in Parkinson's disease (PD) patients offer potentially converging evidence of a PFC-BG mediated gate. PD allows WM to be explored under two important conditions: when "Go" pathway functioning is relatively restored by dopaminergic medication and when the "NoGo" pathway dominates after medication has been withheld. Dopaminergic medication re-balances the interaction of the pathways which, according to theory, allows the WM gate to be opened. However, when medication is withheld, PD patients should be more immune to distraction because of difficulty in initiating a memory update. In a "NoGo" state, the gate is more hesitant to open and should therefore more aptly filter out distractors. In contrast, the difficulty of opening the gate may be disadvantageous for encoding task-relevant information into WM. Consistent with the gating hypothesis, previous research has generally supported impairments in memory for task-relevant information (Gabrieli, Singh, Stebbins, \& Goetz, 1996; Fournet, Moreaud, Roulin, Naegele, \& Pellat, 2000; Lee et al., 2010) and greater distractor resistance (Cools, Miyakawa, Sheridan, \& D'Esposito, 2010; although see Lee et al.) for PD patients who have withheld medication prior to testing or are medication-naïve.

Frontostriatal models of gating have focused on the direct and indirect pathways in the dorsal striatum and its connections with the dorsolateral PFC (Cohen \& Frank, 2009). The connection between the ventromedial PFC and the ventral striatum, however, may also be important for WM. For example, activity in the ventral striatum was correlated with the effects of reward when having to ignore distractors in a WM task, and this region also displayed more connectivity with the ventromedial PFC when performance was rewarded (Fallon \& Cools, 2014). The frontostriatal pathways are roughly organized into four loops in a rostral-caudal gradient and are selective for carrying different types of information (i.e., limbic, associative, sensory, and motor) (Redgrave et al., 2010). Limbic, or affective, information is thought to be carried in the ventral pathway which includes the medial and orbitofrontal PFC, ventral caudate, ventral putamen, and nucleus accumbens. Associative, or cognitive, information is carried in the dorsal pathway and includes the dorsolateral PFC, dorsal caudate, and anterior putamen. The dorsal associative pathway is involved in cognitive control processes such as WM and task switching even when performance is not explicitly rewarded (Braem et al., 2013; McNab \& Klingberg, 2008; Ravizza \& Ciranni, 2002). The current study assesses whether ventral pathways may affect WM performance even when performance is not explicitly rewarded, perhaps via its known involvement in forming stimulusreward associations (O’Doherty et al., 2004).
The ventral pathway is claimed to associate stimuli with value in studies of reinforcement learning (Samanez-Larkin, Worthy, Mata, McClure, \& Knutson, 2014). In WM performance, this process would be important when distraction is present; that is, only task-relevant information should be allowed to enter WM and should be assigned a higher value than distractors. In turn, the dorsal striatum may use this information about value to signal an update of WM. Here we will test for contributions of the dorsal and ventral frontostriatal pathways by manipulating medication status in a group of PD patients. PD primarily affects function of the dorsal striatum leaving the ventral striatum relatively intact in early stages of the disease (Kish, Shannak, \& Hornykiewicz, 1988); however, dopaminergic medication, while restorative to dorsal frontostriatal pathways, "overdoses" the ventral frontostriatal pathways (Cools, Barker, Sahakian, \& Robbins, 2001a; Swainson et al., 2000). With disruption of the ventral system while medicated (ON state), distractors are predicted to be more likely to enter WM because task-relevant information has not been prioritized, i.e., associated with a higher value. When dorsal pathways are disrupted in the OFF state, the gate is less able to update WM with new information; this translates into fewer items altogether being gated into WM with the consequence of lower WM for task-relevant information and the benefit of greater distractor resistance. Thus, disruption of the dorsal frontostriatal pathway should increase resistance to distractors whereas ventral pathway disruption should decrease resistance.

In this paper, we propose a novel way of testing for the contribution of these pathways to WM performance by using a signal detection model. For patients tested in the OFF state, disruption of the "Go" pathway in the dorsal striatum should create difficulty in updating items into WM; thus, there should be fewer items altogether being held in WM. As a consequence, PD patients in the OFF state should be more likely to respond "NO" when asked if a probe item matches an item in WM because it is less likely that the item entered WM. In signal detection terms, this should affect the response criterion (c) with a bias for "NO" responses regardless of whether distraction is present or absent. In contrast, a change in the $d$ ' measure is expected for those in the ON state when the ventral striatum is "overdosed" but only when distraction is present. Prioritizing the encoding of task-relevant information over distractors may be compromised; that is, the faulty ventral pathway may erroneously assign higher value to these distracting items allowing them to enter WM and, consequently, lowering $d^{\prime}$.

In addition to testing WM gating as a function of frontostriatal function, the current study also examines potential commonalities or differences across domains. Verbal, object, and spatial WM studies have reported both common and dissociable patterns of neural engagement using fMRI. Recent studies have shown that sensory and perceptual regions in the 
posterior cortex support the maintenance of different types of information; that is, regions involved in the perception of information also support its representation in WM (Ranganath, DeGutis, \& D’Esposito, 2004; Serences, Ester, Vogel, \& Awh, 2009). Despite early work in primates that suggested separate regions of the PFC supporting spatial and object WM (Wilson, O'Scalaidhe, \& Goldman-Rakic, 1993), recent imaging and patient studies in humans have provided evidence for a more domain-general model of functional organization in the prefrontal executive system (Barbey, Koenigs, \& Grafman, 2013; Chein, Moore, \& Conway, 2011; Fedorenko, Duncan, \& Kanwisher, 2013; Li, Christ, \& Cowan, 2014; although see Nee et al., 2013). For example, one study of complex WM span showed highly overlapping patterns of activity in the PFC for verbal and spatial tasks (Chein et al.). Thus, the literature focusing on executive function in the PFC suggests that the BG-PFC pathways should operate similarly across domains.

Past research does not provide a clear answer as to whether the potential BG-PFC gating mechanism is domain-specific. In simple span tasks, there is some evidence that PD patients have impaired spatial WM (Ventre-Dominey, Bourret, Mollion, Broussolle, \& Dominey, 2014) while verbal WM is relatively intact (Graceffa, Carlesimo, Peppe, \& Caltagirone, 1999). A meta-analysis of WM studies also indicated that spatial span is more severely disrupted in PD than verbal span (Siegert, Weatherall, Taylor, \& Abernethy, 2008). This metaanalysis also reported more domain-general effects when examining complex WM span, and the authors suggest that domain-specificity in simple span tasks may be apparent because spatial tasks require a greater degree of executive processing than verbal tasks given their greater inherent difficulty (Siegert et al.). If both types of information are equally difficult to gate into WM, performance should be affected in both verbal and spatial WM tasks.

The present study uses PD as model to assess the involvement of the BG-PFC pathways in WM across verbal and spatial domains. Toward this end, control participants and PD patients, both ON and OFF dopaminergic medication, were tested in a WM paradigm. WM for task-relevant information at low and high loads were assessed in addition to low load with distraction. The high-load condition was included to increase the difficulty of the WM task comparable to the increase in difficulty produced by presenting distracting information. In this way, we can observe the effects of dorsal and ventral frontostriatal disruption on WM for task-relevant information and vulnerability to distraction. It is predicted that PD patients tested under withdrawal from their medication will show a greater response bias to say "NO" across all conditions. In contrast, patients tested while on their medication are expected to show lower $d^{\prime}$ only in the distraction condition. Further, we predict a domain-general effect, showing the same pattern of results in both verbal and spatial domains.

\section{Materials and methods}

\section{Participants}

Twenty-one patients with idiopathic PD participated in the present study. The patients were recruited from the Movement Disorders Clinic at Michigan State University. All patients were on dopaminergic pharmacological treatment and were in the mild-to-moderate stages of the disease, with Hoehn and Yahr stages ranging from 1 to $3(M=1.63, S D=$ 0.62 ). Twenty-four healthy controls were also recruited from the community. All participants were prescreened based on their neurological and psychiatric history (i.e., no diagnosis of any neurological or psychiatric disorder for which they take medication) and having normal or corrected-to-normal vision. Additional exclusions were made during testing: participants were required to score above 23 on the Mini-Mental State Exam (MMSE) and in the minimal range (i.e., 0-13) for depression on the Beck Depression Inventory II (BDI-II). Based on these criteria, a total of two PD patients and three controls were tested but later excluded. After these exclusions, the control and patient groups were no longer matched for age or the ratio of males to females and, consequently, data from the two oldest female controls were dropped from the analysis. The resulting 19 control participants and 19 PD patients did not statistically differ in age, years of education, or gender ratio (Table 1 ). Both $\mathrm{PD}$ patients and controls were paid US $\$ 10 / \mathrm{h}$ for their participation in the study.

\section{Procedure}

Testing was completed over two sessions approximately 1 week apart for all participants. For controls, both testing sessions were the same. For PD patients, one session was completed on medication, and the other was conducted a minimum of $12 \mathrm{~h}$ from their last dose of dopaminergic medication. To reduce possible order effects, medication status and condition (verbal, spatial) were counterbalanced across participants such that similar numbers of participants were tested first in the OFF or ON stages and in the verbal or spatial condition.

All testing sessions were conducted in the morning. This allowed for the majority of OFF-medication hours for PD patients to occur during sleep. On the day of testing, PD patients either withheld their first dosage or were tested shortly after their first dosage.

Testing was conducted on a laptop computer either in a lab at Michigan State University or in the homes of the PD patients. The testing location remained constant for both sessions. During the testing session, demographic information was collected and the prescreening tests were conducted. All participants were given the Digit Span forward subtest of the Wechsler Adult Intelligence Test (IV) to assess each group's WM capacity. The prescreen tests were completed while PD 
Table 1 Demographic information for patients with Parkinson's disease (PD) and group means and standard deviations for both PD patients and controls

\begin{tabular}{|c|c|c|c|c|c|c|c|c|}
\hline ID & Gender & Age & Edu. & $\mathrm{H} \& \mathrm{Y}$ & BDI-II & MMSE & DSpan & Medications \\
\hline 1 & M & 64 & 19 & 1 & 2 & 29 & 10 & Rasagiline; ropinirole; amantadine \\
\hline 2 & $\mathrm{~F}$ & 66 & 14 & 1 & 5 & 30 & 16 & Carbodopa/levodopa; pramipexole \\
\hline 3 & M & 69 & 12 & 2 & 10 & 30 & 13 & Carbodopa/levodopa \\
\hline 4 & $\mathrm{~F}$ & 63 & 18 & 2 & 8 & 25 & 11 & Rasagiline; carbodopa/levodopa; pramipexole \\
\hline 5 & M & 69 & 14 & 2 & 10 & 25 & 10 & Carbodopa/levodopa \\
\hline 6 & M & 76 & 14 & $N / A$ & 10 & 27 & 10 & Rasagiline; carbodopa/levodopa \\
\hline 7 & $\mathrm{~F}$ & 67 & 24 & 2 & 0 & 30 & 12 & Rasagiline \\
\hline 8 & M & 62 & 19 & 1 & 2 & 30 & 13 & Rasagiline; carbodopa/levodopa \\
\hline 9 & $\mathrm{~F}$ & 57 & 18 & 1 & 1 & 30 & 12 & Rasagiline \\
\hline 10 & M & 56 & 15 & 1 & 5 & 29 & 12 & Rasagiline \\
\hline 11 & $\mathrm{~F}$ & 56 & 25 & 2 & 0 & 30 & 8 & Rasagiline; carbodopa/levodopa; pramipexole \\
\hline 12 & M & 64 & 16 & $N / A$ & 6 & 27 & 11 & Rasagiline; carbodopa/levodopa \\
\hline 13 & $\mathrm{~F}$ & 70 & 12 & 2 & 7 & 28 & 12 & Rasagiline; carbodopa/levodopa \\
\hline 14 & M & 55 & 13 & 2 & 6 & 28 & 13 & Carbodopa/levodopa; ropinirole \\
\hline 15 & M & 65 & 13 & $N / A$ & 1 & 26 & 11 & Carbodopa/levodopa; amantadine \\
\hline 16 & M & 43 & 12 & 1 & 2 & 28 & 9 & Pramipexole \\
\hline 17 & M & 71 & 12 & 2 & 1 & 29 & 13 & Rasagiline; carbodopa/levodopa \\
\hline 18 & M & 75 & 16 & 3 & 2 & 30 & 11 & Rasagiline; carbodopa/levodopa \\
\hline 19 & \multicolumn{8}{|c|}{ PD patients } \\
\hline$M$ & & 63.89 & 15.74 & 1.63 & 4.42 & 28.37 & $11.58^{*}$ & Males: $\mathrm{n}=13$ \\
\hline$S D$ & & 7.89 & 3.90 & 0.62 & 3.49 & 1.71 & 1.80 & Females: $\mathrm{n}=6$ \\
\hline & \multicolumn{8}{|c|}{ Controls } \\
\hline$M$ & & 66.05 & 16.00 & $N / A$ & 5.84 & 27.63 & $9.63 *$ & Males: $\mathrm{n}=12$ \\
\hline$S D$ & & 9.14 & 3.35 & $N / A$ & 3.30 & 2.24 & 1.92 & Females: $\mathrm{n}=7$ \\
\hline
\end{tabular}

$E d u$. Years of education, $H \& Y$ Hoehn and Yahr scale, BDI-II Beck Depression Inventory II, MMSE Mini Mental State Exam, DSpan Digit Spawn

$\mathrm{H} \& \mathrm{Y}$ scales could not be obtained for three patients, as denoted by $N / A$

* Significant difference between patients and controls at $p<.05$

patients were ON medication and was counterbalanced for controls. The majority of the testing session consisted of the verbal and spatial WM tasks.

Task The main task, summarized visually in Fig. 1, consisted of a cross-modal WM task with verbal and spatial components. The spatial task was adapted from McNab and Klingberg (2008) and was translated into the verbal domain to match the spatial task as much as possible.

In the spatial task, participants were asked to memorize the location of dots. The task had three conditions: low-load, high-load, and distraction. In the low-load condition, participants were presented with three red target dots. In the highload, five red target dots were presented. For the distraction condition, three red target dots were shown as well as two yellow distractor dots. There were 12 potential positions where a dot could be displayed; these potential positions formed a rectangle. Each potential location was outlined with a white square. Therefore, while the dots were presented, 12 white squares were also displayed, some of which contained a colored dot. The square each dot was presented in was randomly determined. The dots and squares were presented for 1 , $000 \mathrm{~ms}$, and then there was a maintenance period of 2,000 ms where a white fixation cross was shown. Lastly, a probe was presented for test. Here, the squares were again displayed but now with no colored dots. Instead, one square had a white question mark (?) inside. If the probe matched a location of a red target dot, the correct response was "YES." If, however, the probe matched the location of a yellow distractor dot or was a location where no dot was presented, the correct response was "NO." Answers were submitted using the " 0 " and "." keys on the number pad, such that the two response keys were adjacent to one another. The keys were labeled "YES" and "NO," respectively. In the low-load and highload conditions, there was a $50 \%$ chance the probe would match (i.e., "YES" response) and a $50 \%$ chance the probe would not match (i.e., "NO" response). In the distraction condition, there was a $40 \%$ chance the response should be "YES" 


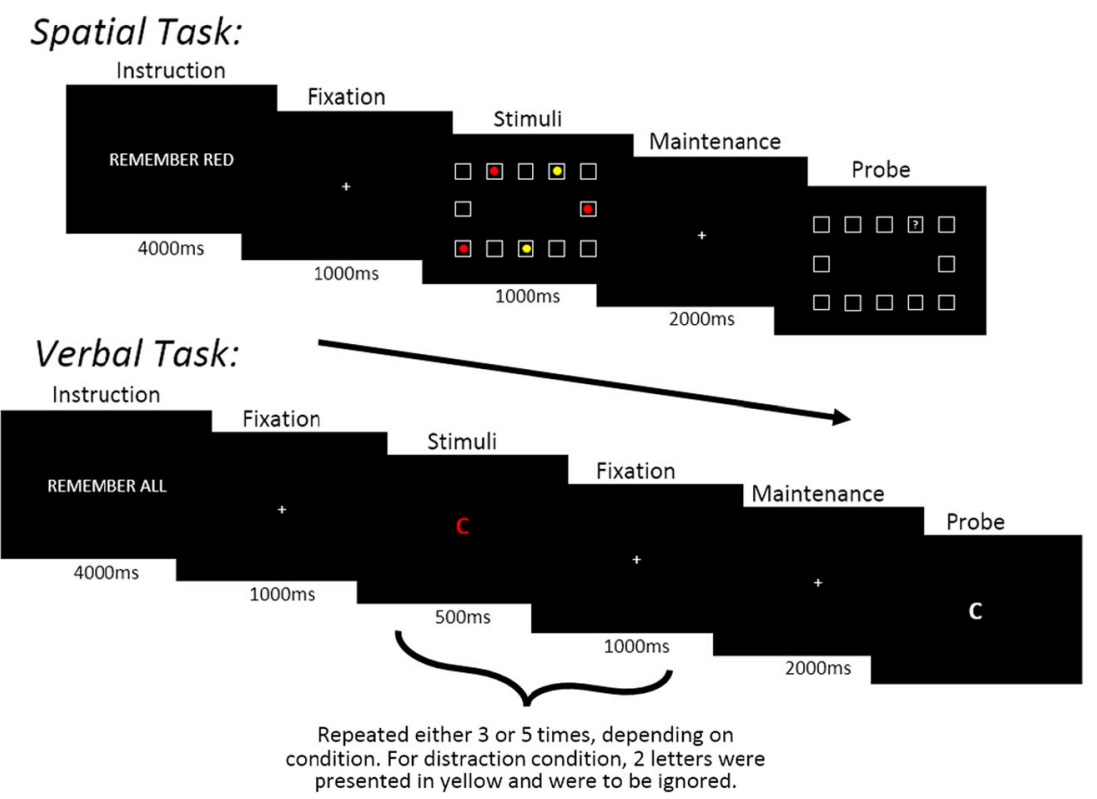

Fig. 1 Timing schedule for both the spatial and verbal working memory tasks

and a $60 \%$ chance the response should be "NO." We slightly increased the "NO" responses in this condition so that we would have enough trials to differentiate between novel nonmatching probes and probes that matched a distracting item. For non-matching probes, there was a $2 / 5$ chance that the probe matched a distractor and a $3 / 5$ chance the probe matched a target letter. Throughout the entirety of the spatial task, there were 60 distractor trials, 40 low-load, and 40 highload that were presented in a random order. Therefore, $54 \%$ of trials had a correct response of "NO," and the remaining $46 \%$ had a correct response of "YES." 1

In the verbal task, participants were asked to memorize a series of letters. The letters were presented sequentially; each was shown for $500 \mathrm{~ms}$ in the center of the screen and then was replaced with a white fixation cross for $1,000 \mathrm{~ms}$, followed by the next letter. As in the spatial task, there were three conditions. In the low-load condition, participants were presented with three red target letters to memorize. In the high-load, five red target letters were shown. For the distraction condition, the participant was to memorize three red target letters but was to ignore two yellow letters that were also presented in the sequence. The order of targets and distractors was random. After the final letter, there was a 2,000 ms maintenance period where again a white fixation cross was presented. Lastly, a probe letter was shown in white until the participant provided a response. If the probe letter matched a red target letter, the

\footnotetext{
${ }^{1}$ A one-sample t-test on response criterion collapsed across domain and condition indicated that there was no significant difference between the controls' mean and an unbiased value of $0, t(18)=0.26, p=.801$. Therefore, the slightly greater quantity of "NO" trials did not cause an overall "NO" bias, and any response biases can instead be attributed to experimental conditions.
}

correct response was "YES." If, however, the probe matched a yellow distractor letter or was a letter that was never presented, the correct response was "NO." Again, answers were submitted using the "0" and "." keys on the number pad. The number of trials and proportion of "YES" and "NO" responses and probe types was equivalent to the spatial task.

Prior to each trial in both domains, the participant was informed whether the trial would contain distractors or not. If the trial contained only red target stimuli (i.e., low-load or high-load condition), "REMEMBER ALL" was displayed on screen in white text for $4,000 \mathrm{~ms}$. If the trial was a distraction condition, "REMEMBER RED" was shown in white text for 4,000 ms. This instruction was followed by a white fixation cross for $1,000 \mathrm{~ms}$, and then the stimuli were presented.

\section{Data analysis}

Due to an overall ceiling effect in accuracy, an edge correction transformation was applied to the dataset. We used the loglinear rule (Hautus, 1995) where the frequency of hit, miss, false alarm, and correct rejection trials for each condition of interest in each participant was calculated and 0.5 was added to each of the four counts for each trial type. Then, hit rates and false alarm rates were calculated in the typical manner.

We used the Gaussian equal variance model from signal detection theory to both calculate a measure of accuracy and a measure of response bias. Accuracy was assessed with $d^{\prime}, d^{\prime}=$ $z(H)-z(F A)$ where $z(\bullet)$ is the inverse of the standard normal cumulative distribution function and $H$ and $F A$ are the hit and false alarm rates, respectively. Response bias was measured with the location of the criterion, $c=-.5[z(H)+z(F A)]$. Positive values of $c$ indicate a bias to say "NO" and negative 
values a bias to say "YES." For controls, the $d$ ' and $c$ scores were then averaged across the two sessions.

Trials with slow responses were removed from analyses. Specifically, each individual participant's mean response time (RT) was calculated, and trials where the RT was three standard deviations (SDs) above the participant's individual mean were removed. As a result, $1.77 \%$ of all trials were excluded: $1.68 \%$ were removed from the controls over both sessions, $2.11 \%$ from the PD patients OFF medication, and $1.62 \%$ from the PD patients ON medication.

\section{Results}

In the first analysis, the performance of patients in the OFF state was compared to that of them in the $\mathrm{ON}$ state. A nice feature of this comparison is that PD patients act as their own control without potential confounds of differences in demographic variables which is always a potential problem when using between-group comparisons. Dopaminergic medication should restore functioning of the dorsal frontostriatal pathways which facilitates movement initiation and, perhaps, the updating of WM with new items - both relevant and irrelevant. Conversely, patients in the OFF state should have greater difficulty updating the contents of WM. If items are simply not present in WM, this should be observed in a change of response criterion with those in the OFF state showing a bias to respond "NO" when asked to recognize whether the probe was part of the study set. In contrast, the same patients tested in the ON state should show no bias in $c$, but $d^{\prime}$ should be impaired in the distraction condition. In this case, overdosing the ventral striatum should cause impairments in associating higher value with task-relevant information than irrelevant information.

\section{Within-subject analyses}

Response criterion A 2 (Medication Status: ON; OFF) $\times 3$ (Condition: High-load; Low-load; Distraction) $\times 2$ (Domain: Verbal; Spatial) repeated measures (RM) ANOVA was run on the response criteria, $c$, and only the main effect of Medication Status was significant, $F(1,18)=6.41, p=.021, \eta_{\mathrm{p}}{ }^{2}=.263$ (see Fig. 2). Patients OFF medication showed a more conservative response bias such that they were more likely to respond "NO" to probes across all conditions than when they were $\mathrm{ON}$ medication.

d' analysis A 2 (Medication Status: ON; OFF) $\times 3$ (Condition: High-load; Low-load; Distraction) $\times 2$ (Domain: Verbal; Spatial) RM ANOVA on $d^{\prime}$ scores was used. There was a significant main effect of Condition, $F(2,17)=20.56, p<$ $.001, \eta_{\mathrm{p}}^{2}=.533$, indicating that performance was worst in the high-load condition, and performance in the distraction condition trended to be best (distraction vs. low-load: $t(37)=$ $2.00, p=.053$; low-load vs high-load: $t(37)=5.35, p<.001$; distraction vs. high-load: $t(37)=6.13, p<.001)$.

The interaction of Medication Status $\times$ Condition, was significant, $F(2,17)=7.73, p=.002, \eta_{\mathrm{p}}{ }^{2}=.300$, indicating that Medication Status affected performance only in the distraction condition (paired t-tests comparing OFF vs. ON in distraction condition: $t(18)=2.94, p=.009$; low-load: $t(18)=0.81, p=$ .428 ; high-load: $t(18)=0.46, p=.648)$. Patients who were on their medication were more vulnerable to distraction than those withholding their medication. This interaction was not mediated by Domain (Medication Status $\times$ Condition $\times$ Domain interaction, $\left.F(2,17)=0.76, p=.473, \eta_{\mathrm{p}}{ }^{2}=.041\right)$, and inspection of Fig. 3 indicates that the pattern of distraction effects was similar in both the verbal and spatial conditions.

Moreover, there was a trending Domain $\times$ Condition interaction, $F(2,17)=2.50, p=.096, \eta_{\mathrm{p}}{ }^{2}=.122$. This was a result of the verbal condition having significantly higher $d^{\prime}$ in the distraction condition, $t(37)=2.40, p=.021$, while the lowand high-load conditions had similar values for $d^{\prime}$ across domains, $t(37)=1.37, p=.180$ and $t(37)=0.76, p=.454$ respectively.

\section{Correct rejection rates to distracting versus novel probes} In the distraction condition, probes that did not match items in the memory set (i.e., correct response of "NO") could either be novel or correspond to one of the yellow distractor items. A 2 (Medication Status: ON; OFF) $\times 2$ (Probe Type: Novel; Yellow Distractor) $\times 2$ (Domain: Verbal; Spatial) RM ANOVA comparing edge-corrected correct rejection rates for these non-matching probe types showed a significant main effect of Probe Type, $F(1,18)=7.15, p=.016, \eta_{\mathrm{p}}{ }^{2}=.284$, indicating that distractor probes were more difficult to reject than novel probes. However, this effect did not interact with Medication Status as the Medication Status $\times$ Probe Type interaction was not significant, $F(1,18)=0.19, p=.670$, $\eta_{\mathrm{p}}{ }^{2}=.010$. Further, the Medication Status $\times$ Probe Type $\times$ Domain interaction was also not significant, $F(1,18)=0.58$, $p=.456, \eta_{\mathrm{p}}{ }^{2}=.031$.

Response time The same RM ANOVA run on $d^{\prime}$ was also applied to RT data for correct responses. Speed of responding to the probe item was not affected by Medication Status, although there was a trend for patients in the OFF state to be slower across all conditions than those in the ON state, $F(1,18)=3.33, p=.085, \eta_{\mathrm{p}}{ }^{2}=.156$. There was a significant main effect of Condition, $F(2,17)=29.20, p<.001, \eta_{\mathrm{p}}{ }^{2}=$ .619 , indicating that the low-load condition elicited the fastest responses, then the distraction condition (low-load vs. distraction: $t(18)=5.38, p<.001$ ), and the high-load condition required the most time (distraction vs. high-load: $t(18)=$ $4.15, p=.001$ ). Additionally, there was a significant main 


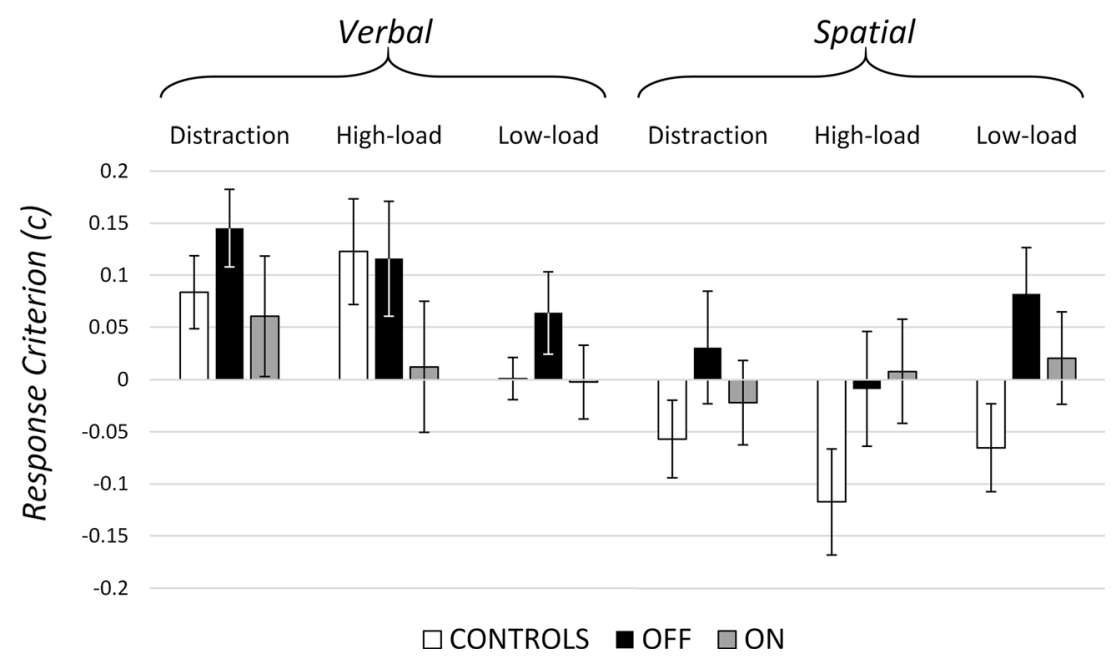

Fig. 2 Means with standard error bars for response criterion, $c$, in all conditions in both domains for all three experimental groups

effect of Domain, $F(1,18)=5.10, p=.037, \eta_{\mathrm{p}}^{2}=.221$, with faster RTs in the spatial condition.

A significant Domain $\times$ Condition interaction, $F(2,17)=$ $6.65, p=.003, \eta_{\mathrm{p}}^{2}=.270$, showed that the low-load condition only trended to have longer RTs in the verbal domain $(t(37)=$ $1.84, p=.073)$; however, both the distraction and high-load conditions had significantly longer RTs in the verbal domain, $t(37)=2.60, p=.013$ and $t(37)=3.47, p=.001$ respectively.

\section{Controls versus ON medication}

To further assess the effects of medication on WM performance, patients ON dopaminergic medication were also compared with non-medicated control subjects. Note that these analyses used mixed factor ANOVAs as control participants and PD patients are separate groups.
Response criterion A 2 (Group: Controls; ON) $\times 3$ (Condition: High-load; Low-load; Distraction) $\times 2$ (Domain: Verbal; Spatial) mixed-factor ANOVA was run on the response criteria, $c$. A main effect of Domain, $F(1,36)=5.09$, $p=.030, \eta_{\mathrm{p}}{ }^{2}=.124$, showed a higher "NO" rate in the verbal domain and a higher "YES" rate in the spatial domain, $t(37)=$ $2.20, p=.034$.

A trend in the Domain $\times$ Group interaction, $F(1,36)=2.85$, $p=.100, \eta_{\mathrm{p}}^{2}=.073$, showed that when collapsed across Condition, controls trended to respond with higher "YES" rates in the spatial domain while both groups responded similarly in the verbal domain (verbal: $t(36)=1.02, p=.314$, spatial: $t(36)=1.78, p=.083)$.

d' analysis A 2 (Group: Controls; ON) $\times 3$ (Condition: Highload; Low-load; Distraction) $\times 2$ (Domain: Verbal; Spatial)

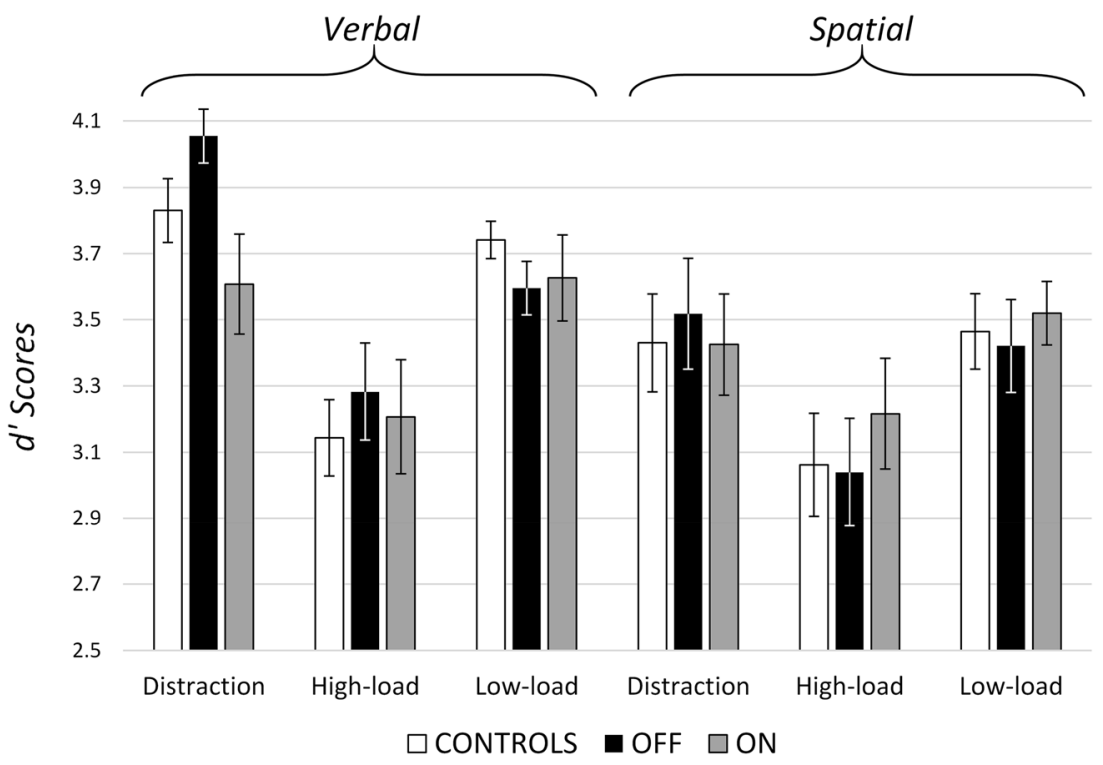

Fig. 3 Means with standard error bars for $d^{\prime}$ scores in all conditions in both domains for all three experimental groups 
mixed-factor ANOVA was run on $d^{\prime}$ scores. This resulted in a main effect of Condition, $F(2,35)=39.00, p<.001, \eta_{\mathrm{p}}{ }^{2}=$ .520 , where both the distraction and low-load condition had higher performance than the high-load condition (distraction vs. high-load: $t(37)=6.40, p<.001$; low-load vs. high-load: $t(37)=7.94, p<.001)$, but the distraction and low-load conditions did not differ $(t(37)=.304, p=.763)$.

Individuals with PD ON medication showed lower distractor resistance compared to controls numerically, but the Group $\times$ Condition interaction was not significant, $F(2,35)=2.06, p=.134, \eta_{\mathrm{p}}{ }^{2}=.054$.

Additionally, there was a trending Domain $\times$ Condition interaction, $F(2,35)=2.52, p=.088, \eta_{\mathrm{p}}{ }^{2}=.065$. This was a result of the verbal condition trending towards higher $d^{\prime}$ in the distraction and low-load conditions (verbal vs. spatial in distraction: $t(37)=1.90, p=.065$; low-load: $t(37)=1.90$, $p=.065)$ while the high-load condition had similar accuracy across domains $(t(37)=0.27, p=.793)$.

\section{Correct rejection rates to distracting versus novel probes}

A 2 (Group: Controls; ON) $\times 2$ (Probe: Yellow; Novel) $\times 2$ (Domain: Verbal; Spatial) mixed-factor ANOVA was run comparing correct rejection rates in the distraction condition. Main effects of Probe Type and Domain were found, $F(1,36)$ $=10.47, p=.003, \eta_{\mathrm{p}}{ }^{2}=.225$ and $F(1,36)=7.40, p=.010$, $\eta_{\mathrm{p}}{ }^{2}=.171$, respectively. Again, responses were more accurate in the verbal domain over the spatial, $t(37)=2.71, p=.010$, and trials with a novel probe rather than a distractor probe had higher correct rejection rates, $t(37)=3.28, p=.002$.

Response time The mixed-factor ANOVA of RTs for correct trials reveals similar findings as our previous results within PD patients. Namely, significant main effects of Domain, $F(1,36)$ $=9.01, p=.005, \eta_{\mathrm{p}}{ }^{2}=.200$, and Condition, $F(2,35)=73.41$, $p<.001, \eta_{\mathrm{p}}{ }^{2}=.671$. Lastly, we found a significant Domain $\times$ Condition interaction, $F(2,35)=9.33, p<.001, \eta_{\mathrm{p}}{ }^{2}=.206$, showing as the domain became more difficult (i.e., verbal) the high-load condition took disproportionately longer than the other two conditions.

\section{Controls versus OFF medication}

To assess the effects of PD on WM performance, patients in the OFF state were compared to controls. Note that these analyses will again use mixed factor RM ANOVAs as control participants and PD patients are separate groups.

Response criterion A 2 (Group: Controls; OFF) $\times 3$ (Condition: High-load; Low-load; Distraction) $\times 2$ (Domain: Verbal; Spatial) mixed-factor ANOVA was run on the response criteria, $c$. PD patients in the OFF state showed a significant bias to respond "NO" across conditions compared to control participants, as witnessed by a main effect of Group,
$F(1,36)=5.28, p=.027, \eta_{\mathrm{p}}{ }^{2}=.128$. A main effect of Domain, $F(1,36)=13.25, p=.001, \eta_{\mathrm{p}}{ }^{2}=.269$, showed a higher "NO" rate in the verbal domain while the spatial domain tended to be relatively unbiased. A significant Domain $\times$ Condition interaction, $F(2,35)=4.13, p=.020, \eta_{\mathrm{p}}{ }^{2}=.103$, showed that the verbal and spatial domains had similar levels of response bias in the low-load condition, $t(37)=0.62, p=.540$, but response bias was significantly more positive (i.e., more likely to say "NO") in the verbal domain for both the distraction and high-load conditions $(t(37)=3.18, p=.003 ; t(37)=3.46, p=.001$, respectively $)$.

d' analysis A 2 (Group: Controls; OFF) $\times 3$ (Condition: Highload; Low-load; Distraction) $\times 2$ (Domain: Verbal; Spatial) mixed-factor ANOVA was run on $d^{\prime}$ scores. This resulted in main effects of Domain, $F(1,36)=8.76, p=.005, \eta_{\mathrm{p}}{ }^{2}=.196$, with significantly better performance found in the verbal domain, $t(37)=3.00, p=.005$, and Condition, $F(2,35)=58.68$, $p<.001, \eta_{\mathrm{p}}{ }^{2}=.620$, where across domains the distraction condition had the highest performance, followed by the lowload condition, and the high-load condition had the lowest accuracy (distraction vs. low-load: $t(37)=3.20, p=.003$; low-load vs. high-load: $t(37)=7.21, p<.001$ ).

The Group $\times$ Condition interaction only trended towards significance, $F(2,35)=2.63, p=.079, \eta_{\mathrm{p}}{ }^{2}=.068$; however, those with PD OFF medication did show better distractor resistance compared to controls numerically.

Additionally, there was a significant Domain $\times$ Condition interaction, $F(2,35)=3.93, p=.024, \eta_{\mathrm{p}}{ }^{2}=.098$. This was a result of the verbal condition having significantly higher $d^{\prime}$ in the distraction and low-load conditions (verbal vs spatial in distraction: $t(37)=4.15, p<.001$; low-load: $t(37)=2.74$, $p=.009)$ while the high-load condition had similar accuracy across domains $(t(37)=1.13, p=.268)$.

\section{Correct rejection rates to distracting versus novel probes} A 2 (Group: Controls; OFF) $\times 2$ (Probe: Yellow; Novel) $\times 2$ (Domain: Verbal; Spatial) mixed-factor ANOVA was run comparing correct rejection rates in the distraction condition. Main effects of Probe Type and Domain were found, $F(1,36)$ $=18.77, p<.001, \eta_{\mathrm{p}}{ }^{2}=.343$ and $F(1,36)=15.36, p<.001$, $\eta_{\mathrm{p}}{ }^{2}=.299$ respectively. Additionally, the Probe Type $\times$ Domain interaction was significant, $F(1,36)=6.14$, $p=.018, \eta_{\mathrm{p}}{ }^{2}=.146$, indicating that yellow probes had much lower accuracy compared to novel probes in the spatial condition, $t(37)=3.42, p=.002$. Yellow probes were also significantly more difficult in the verbal condition, $t(37)=2.36$, $p=.024$, but by a much lesser extent (Fig. 4).

Response time Again, the mixed-factor ANOVA of RTs for correct trials reveals similar findings as our previous results within PD patients. Namely, significant main effects of Domain, $F(1,36)=6.97, p=.012, \eta_{\mathrm{p}}{ }^{2}=.162$, and Condition, $F(2,35)=50.95, p<.001, \eta_{\mathrm{p}}{ }^{2}=.586$. 


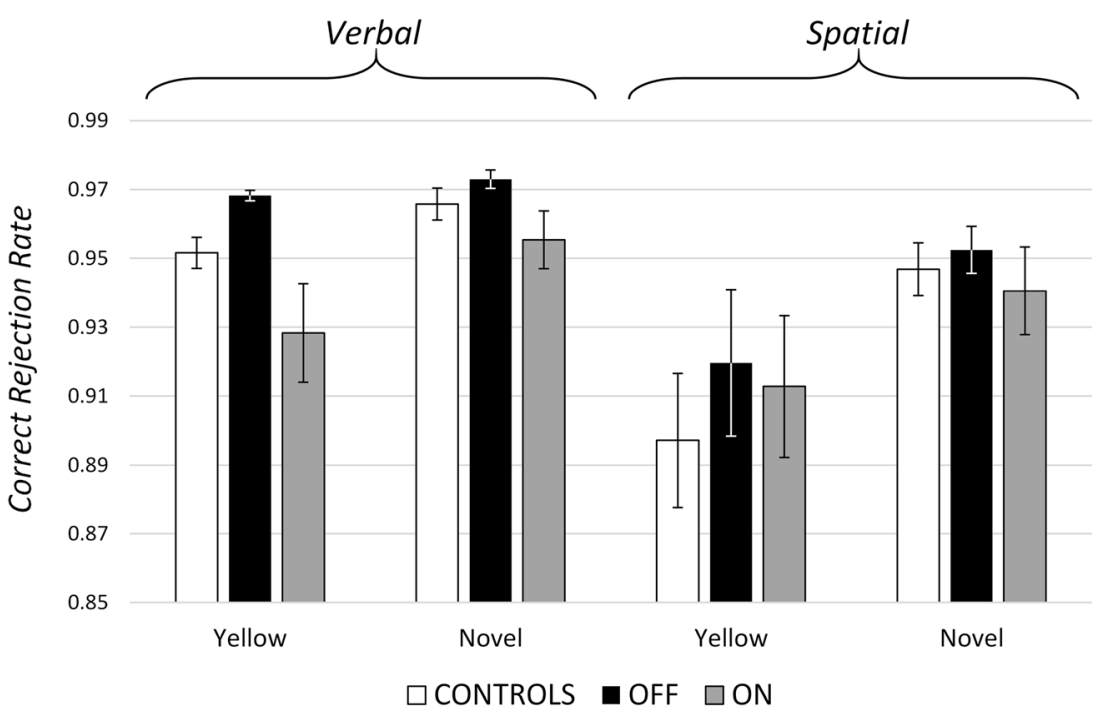

Fig. 4 Means with standard error bars for correct rejection rates of both novel and yellow probes in the distraction condition in both domains for all three experimental groups

Additionally, here we found an expected main effect of Group, $F(1,36)=4.15, p=.049, \eta_{\mathrm{p}}^{2}=.103$, where our PD patients OFF medication had slower RTs than control participants. Lastly, we found a significant Domain $\times$ Condition interaction, $F(2,35)=7.64, p=.001, \eta_{\mathrm{p}}^{2}=.175$, showing that the low-load condition did not significantly vary in RT but instead trended between domains, $t(37)=1.69, p=.099$; however, both the distraction and high-load conditions elicited significantly longer RTs in the verbal domain, $t(37)=2.42, p=.020$ and $t(37)=3.31, p=.002$ respectively (Fig. 5).

\section{Discussion}

The goal of the present study was to more precisely characterize the contribution of frontostriatal pathways to WM gating using signal detection analyses. Under withdrawal from dopaminergic medication, the "NoGo" pathway dominates in the dorsal striatum and increases the difficulty of updating WM for both task-relevant and distracting information. As a consequence, it was expected that fewer items could enter WM, and PD patients in the OFF state would show an increased bias to respond "NO" in a recognition task. We found that medication withdrawal affected response criterion such that a "NO" bias was observed in the OFF state across all conditions and domains in comparison to both healthy controls and when they acted as their own controls in the ON state. These results are consistent with the hypothesis that the dorsal PFC-BG gating mechanism allows or prevents updates of WM.

In contrast, stimulus valuation processes were thought to be impaired by dopaminergic medication which overdoses the

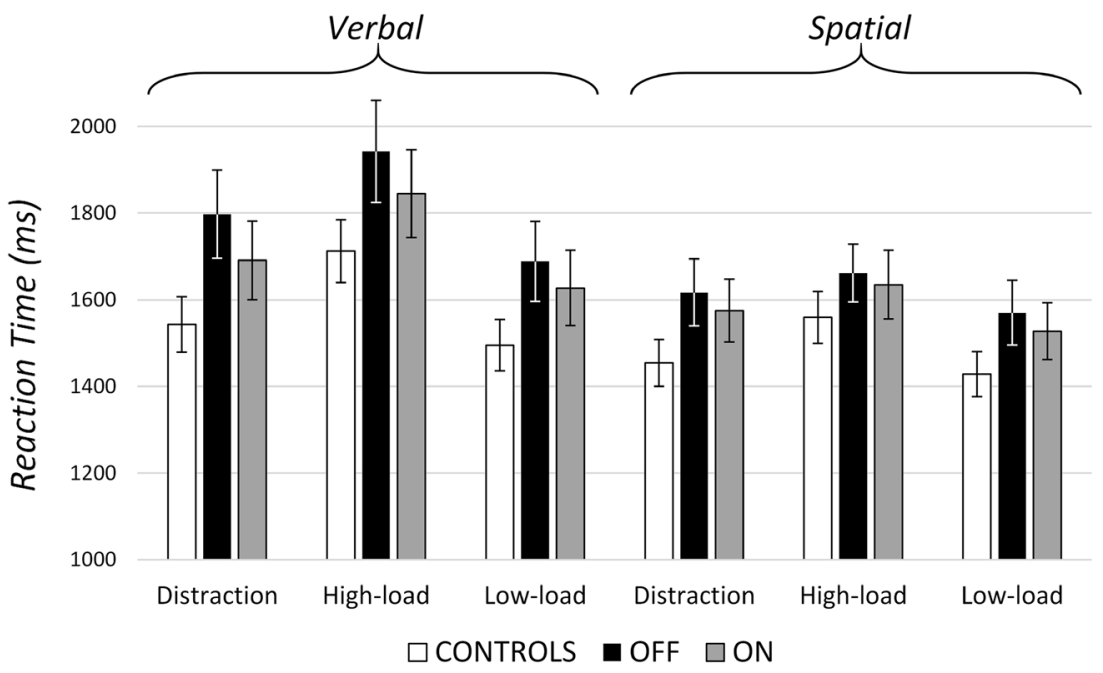

Fig. 5 Means with standard error bars for reaction times in all conditions in both domains for all three experimental groups 
ventral pathway. This was predicted to affect $d^{\prime}$ primarily in the distraction condition. As predicted, medication status affected $d^{\prime}$ in the distraction condition but not in the high- or low-load conditions. This effect, however, may reflect greater distraction resistance in the OFF state or lower distractor resistance in the $\mathrm{ON}$ state; although $d^{\prime}$ differed as a function of medication status, $d^{\prime}$ was not significantly lower or higher in PD compared to healthy controls. The lack of differences between controls and patients may be due to the high $d^{\prime}$ estimates in this study. Estimates of $d^{\prime}$ were near ceiling and may have obscured potential differences in this measure. Thus, although we found evidence for an impairment in $d^{\prime}$ when comparing patients in the ON and OFF state, we cannot associate this with impaired functioning of the dorsal pathway, the ventral pathway, or both. Understanding the mechanism producing this difference in $d^{\prime}$ is an avenue for further research.

Our use of response criterion to assess the viability of the gating hypothesis does not presume that this is a pure measure of gating. A change in the response criterion could also be caused by perseverative responding which is a known marker of PFC pathology (Milner, 1963). It is unclear, however, why "NO" actions would be more prevalent than "YES" actions if this were the case. Another possibility is that the decision criterion changes for reporting a target and, in effect, becomes more conservative when dopaminergic medication is withdrawn. For example, one study found that PD patients with apathy made more conservative decisions than those without apathy (Martínez-Horta et al., 2014), and the PFC-BG are known to be involved in motivational behaviors (Pessoa, 2009). Thus, the change in the prevalence of "NO" responses is consistent with an impaired WM updating mechanism, but further studies are necessary to determine whether this is due to gating or another factor such as motivation which can also affect WM performance (Beck, Locke, Savine, Jimura, \& Braver, 2010; Gilbert \& Fiez, 2004; Krawczyk, Gazzaley, \& D'Esposito, 2007).

In our WM task, PD patients OFF medication were more resistant to distraction than when tested ON medication. In a task switching study, however, PD patients tested under medication withdrawal were slower to switch when distractors were present than those tested in the ON state (Cools, Barker, Sahakian, \& Robbins, 2001a). One explanation for this difference may be that distraction has separate effects on performance if it is based on a memory representation or a stimulus that is present. Distractors may not be gated into memory but still have an effect when having to act on stimuli that are present in the environment. In addition, the type of distractor may be important; in particular, distractors that afford an incongruent response increase response selection demands that are known to tap functions of the dorsal striatum (Barber \& Carter, 2004; Cools, Barker, Sahakian, \& Robbins, 2001a, b; Ravizza \& Ciranni, 2002; Sylvester et al., 2003). The incongruent actions afforded by a stimulus that must be updated or ignored may be less salient than two incongruent motor responses.

Most models of WM posit domain-specific subsystems that are dedicated to maintaining information in phonological, semantic, feature, object, and spatial codes. These models suggest that executive processes determine which information is selected, maintained, and discarded from these domainspecific subsystems (Anderson, 1993; Baddeley \& Hitch, 1974; Brown, Preece, \& Hulme, 2000; Cowan, 1999; Farrell \& Lewandowsky, 2002; Monsell, 1984). Our results (for both $d^{\prime}$ and $c$ ) are consistent with the hypothesis that the BG-PFC filter both spatial and verbal information before it is allowed entrance into the WM system and, thus, seems to be part of the central executive component of WM. Previous studies (e.g., Baier et al., 2010; Cools et al., 2010; McNab \& Klingberg, 2008) have shown similar effects using various methodologies; however, our results provide more direct support for a PFC-BG gating mechanism across multiple domains, as we found similar effects in both spatial and verbal WM tasks.

The spatial domain has been shown to have a more pronounced impairment in WM over the verbal domain in PD patients (Siegert et al., 2008). Although we found support for a domain-general impairment in WM, it is possible that dissociations between verbal and spatial WM reflect differences in the progress of the disease. In PD, dopamine cell death proceeds from the lateral tier of the substantia nigra (SNI) to medial nigral component (SNm) (Duke, Moran, Pearce, \& Graeber, 2007). If spatial WM involves more dorsolateral parts of striatum than verbal WM, then the presence of domainspecific impairments might vary with disease progression. For example, Owen, Iddon, Hodges, Summers, and Robbins (1997) found selective deficits to spatial WM in early stages of PD. However, we found the opposite result as would be predicted by disease progression; namely, the strongest differences between groups were found in the verbal WM task rather than the spatial WM task. Nevertheless, it is possible that some of the spatial stimuli were coded verbally which may have diminished effects between domain. The short encoding time and number of locations should have made this more difficult than remembering the memoranda spatially. Thus, although there was a relative difference between verbal and spatial conditions, the difference may not have been absolute which may have diminished possible domain effects.

While the present study has provided support for the BGmediated gating model, much still remains unknown about precisely when and how gating takes place. For example, recent evidence has shown that distractor resistance during encoding may rely on separable processes versus when distracting information is presented during a delay interval, as performance in these two conditions is not strongly correlated (McNab \& Dolan, 2014). It is possible that there are separable corticostriatal loops or a different neural mechanism altogether that filters distraction during encoding and 
maintenance. Similar to the current study, previous studies finding support for PFC-BG mediated gating have all presented distractors during encoding (Blatt, Vellage, Baier, \& Müller, 2014; Lee et al., 2010; McNab \& Klingberg, 2008; Baier et al., 2010) or both encoding and maintenance (Cools et al., 2010). It is possible that PFC-BG gating is primarily important when WM must be updated with task-relevant items rather than when distractors are presented alone during a delay interval. Some evidence for this comes from a study by Fallon and Cools (2014) who found more activity in the dorsal striatum when WM must be updated with items presented during the delay than when those items were to be ignored. Future research could address whether similar or dissociable neural mechanisms gate distractors at different stages of WM.

We have primarily discussed a BG-PFC gate from the viewpoint of encoding information into WM; however, it is also possible that differences in performance are due to gating processes at retrieval. In fact, the results of one study suggest that corticostriatal loops are more critical for output gating in which items are selected from WM than input gating (Chatham, Frank, \& Badre, 2014). In that study, PFC-BG connectivity was highest when a cue indicating the relevant items in the memory set was delivered after the memory set rather than before (Chatham et al.). Thus, a better understanding of PFC-BG functioning at different stages may also shed light onto the WM gate.

PD patients are an informative group to test hypotheses about the necessity of dopaminergic processes in frontostriatal pathways. Neuroimaging studies have suggested this system is important for WM gating and this predicts that PD patients in the OFF state should show impairments of WM (Fallon \& Cools, 2014; McNab \& Klingberg, 2008). Our results showing a change in response criterion support this hypothesis using a converging method. While supportive, this study alone cannot definitively tie WM performance with impairments of this system, given that other neurological abnormalities are associated with PD outside of the dopaminergic frontostriatal pathways. Thus, PD is not a perfect model of frontostriatal functioning; however, impairments predicted to be the result of frontostriatal function should logically be observed in this group as a first step.

In conclusion, PD patients OFF their medication showed a bias to reject probes as matching the contents of WM, such that they missed probes that did match encoded items and correctly rejected distractors. This is consistent with the hypothesis that the dorsal PFC-BG provides a signal to update WM. In contrast, PD patients showed lower sensitivity in discriminating between task-relevant and distracting information when tested ON their medication. We suggest that this difference is due to an overdose of the ventral pathways in stimulus valuation processes; however, further work is necessary to establish whether this difference was due to greater resistance in the OFF state, lower resistance in the ON state, or both. Finally, our results are consistent with the idea that the
BG-PFC acts as a domain-general gate because performance was similar across verbal and spatial domains.

Acknowledgments We would like to thank Dr. John Goudreau for his help in clinically assessing our Parkinson's patients and Doozie Russell for her assistance recruiting Parkinson's patients. We would also like to thank Elyse Horak for her help in recruiting and testing control subjects and Shawn Ell for helpful discussions.

\section{Compliance with ethical standards}

Funding This study was generously funded by the National Science Foundation Early Development CAREER award (\#1149078) to S.R.

\section{References}

Anderson, J. R. (1993). Rules of the mind. Hillsdale, NJ: Erlbaum.

Baddeley, A. D., \& Hitch, G. J. (1974). Working memory. In G. H. Bower (Ed.), The psychology of learning and motivation: Advances in research and theory (Vol. 8, pp. 47-90). New York: Academic Press.

Baier, B., Karnath, H., Dieterich, M., Birklein, F., Heinze, C., \& Muller, N. G. (2010). Keeping memory clear and stable-The contribution of human basal ganglia and prefrontal cortex to working memory. The Journal of Neuroscience, 30(29), 9788-9792.

Barber, A. D., \& Carter, C. S. (2004). Cognitive control involved in overcoming prepotent response tendencies and switching between tasks. Cerebral Cortex, 15(7), 899-912.

Barbey, A. K., Koenigs, M., \& Grafman, J. (2013). Dorsolateral prefrontal contributions to human working memory. Cortex, 49(5), 11951205.

Beck, S. M., Locke, H. S., Savine, A. C., Jimura, K., \& Braver, T. S. (2010). Primary and secondary rewards differentially modulate neural activity dynamics during working memory. PLoS ONE, 5(2), 113.

Blatt, J., Vellage, A., Baier, B., \& Müller, N. G. (2014). The contribution of acetylcholine and dopamine to subprocesses of visual working memory-What patients with amnestic mild cognitive impairment and Parkinson's disease can tell us. Neuropsychologia, 61, 89-95.

Braem, S., King, J. A., Korb, F. M., Krebs, R. M., Notebaert, W., \& Egner, T. (2013). Affective modulation of cognitive control is determined by performance-contingency and mediated by ventromedial prefrontal and cingulate cortex. The Journal of Neuroscience, 33(43), 16961-16970.

Brown, G. D., Preece, T., \& Hulme, C. (2000). Oscillator-based memory for serial order. Psychological Review, 107(1), 127-181.

Chatham, C. H., Frank, M. J., \& Badre, D. (2014). Corticostriatal output gating during selection from working memory. Neuron, 81(4), 930 942.

Chein, J. M., \& Fiez, J. A. (2010). Evaluating models of working memory through the effects of concurrent irrelevant information. Journal of Experimental Psychology: General, 139(1), 117-137.

Chein, J. M., Moore, A. B., \& Conway, A. R. A. (2011). Domain-general mechanisms of complex working memory span. NeuroImage, 54(1), 550-559.

Chen, S. H., \& Desmond, J. E. (2005). Temporal dynamics of cerebrocerebellar network recruitment during a cognitive task. Neuropsychologia, 43(9), 1227-1237. 
Cohen, M. X., \& Frank, M. J. (2009). Neurocomputational models of basal ganglia function in learning, memory and choice. Behavioural Brain Research, 199(1), 141-156.

Cools, R., Barker, R. A., Sahakian, B. J., \& Robbins, T. W. (2001a). Enhanced or impaired cognitive function in Parkinson's disease as a function of dopaminergic medication and task demands. Cerebral Cortex, 11(12), 1136-1143.

Cools, R., Barker, R. A., Sahakian, B. J., \& Robbins, T. W. (2001b). Mechanisms of cognitive set flexibility in Parkinson's disease. Brain, 124(12), 2503-2512.

Cools, R., Miyakawa, A., Sheridan, M., \& D’Esposito, M. (2010). Enhanced frontal function in Parkinson's disease. Brain, 133(1), 225-233.

Cowan, N. (1999). An embedded-processes model of working memory. In A. Miyake \& P. Shah (Eds.), Models of working memory: Mechanisms of active maintenance and executive control (pp. 63101). New York: Cambridge University Press.

Duke, D. C., Moran, L. B., Pearce, R. K. B., \& Graeber, M. B. (2007). The medial and lateral substantia nigra in Parkinson's disease: mRNA profiles associated with higher brain tissue vulnerability. Neurogenetics, 8(2), 83-94.

Fallon, S. J., \& Cools, R. (2014). Reward acts on the pFC to enhance distractor resistance of working memory representations. Journal of Cognitive Neuroscience, 26(12), 2812-2826.

Farrell, S., \& Lewandowsky, S. (2002). An endogenous distributed model of ordering in serial recall. Psychonomic Bulletin \& Review, 9(1), 59-79.

Fedorenko, E., Duncan, J., \& Kanwisher, N. (2013). Broad domaingenerality in focal regions of frontal and parietal cortex. Proceedings of the National Academy of Sciences, 110(41), 16616-16621.

Fournet, N., Moreaud, O., Roulin, J. L., Naegele, B., \& Pellat, J. (2000). Working memory functioning in medicated Parkinson's disease patients and the effect of withdrawal of dopaminergic medication. Neuropsychology, 14(2), 247-253.

Frank, M. J., Loughry, B., \& O’Reilly, R. C. (2001). Interactions between frontal cortex and basal ganglia in working memory: A computation model. Cognitive, Affective, \& Behavioral Neuroscience, 1(2), 137-160.

Gabrieli, J. D. E., Singh, J., Stebbins, G. T., \& Goetz, C. G. (1996). Reduced working memory span in Parkinson's disease: Evidence for the role of frontostriatal system in working and strategic memory. Neuropsychology, 10(3), 322-332.

Gilbert, A. M., \& Fiez, J. A. (2004). Integrating reward and cognition in the frontal cortex. Cognitive, Affective, and Behavioral Neuroscience, 4(4), 540-552.

Graceffa, A. M., Carlesimo, G. A., Peppe, A., \& Caltagirone, C. (1999). Verbal working memory deficit in Parkinson's disease subjects. European Neurology, 42(2), 90-94.

Hautus, M. J. (1995). Corrections for extreme proportions and their biasing effects on estimated values of $d^{\prime}$. Behavior Research Methods, Instruments, \& Computers, 27(1), 46-51.

Kish, S. J., Shannak, K., \& Hornykiewicz, O. (1988). Uneven pattern of dopamine loss in the striatum of patients with idiopathic Parkinson's disease. Pathophysiologic and clinical implications. New England Journal of Medicine, 318(14), 876-880.

Krawczyk, D. C., Gazzaley, A., \& D’Esposito, M. (2007). Reward modulation of prefrontal and visual association cortex during an incentive working memory task. Brain Research, 1141, 168-177.

Lee, E.-Y., Cowan, N., Vogel, E. K., Rolan, T., Valle-Inclán, F., \& Hackley, S. A. (2010). Visual working memory deficits in patients with Parkinson's disease are due to both reduced storage capacity and impaired ability to filter out irrelevant information. Brain, 133(9), 2677-2689.

Li, D., Christ, S. E., \& Cowan, N. (2014). Domain-general and domainspecific functional networks in working memory. NeuroImage, 102(Pt 2), 646-656.
Martínez-Horta, S., Riba, J., de Bobadilla, R. F., Pagonabarraga, J., Pascual-Sedano, B., Antonijoan, R. M., Romero, S., Mañanas, M. À., García-Sanchez, C., \& Kulisevsky, J. (2014). Apathy in Parkinson's disease: Neurophysiological evidence of impaired incentive processing. Journal of Neuroscience, 34(17), 5918-5926.

McNab, F., \& Dolan, R. J. (2014). Dissociating distractor-filtering at encoding and during maintenance. Journal of Experimental Psychology: Human Perception and Performance, 40(3), 960-967.

McNab, F., \& Klingberg, T. (2008). Prefrontal cortex and basal ganglia control access to working memory. Nature Neuroscience, 11(1), 103-107.

Miller, E. K., \& Cohen, J. D. (2001). An integrative theory of prefrontal cortex function. Annual Review of Neuroscience, 24, 167-202.

Milner, B. (1963). Effects of different brain lesions on card sorting. Archives of Neurology, 9(1), 90-100.

Monsell, S. (1984). Components of working memory underlying verbal skills: A "distributed capacities" view. In H. Bouma \& D. G. Bouwhuis (Eds.), Attention and performance $X$ (pp. 327-350). London: Erlbaum.

Moore, A. B., Li, Z., Tyner, C. E., Hu, X., \& Crossom, B. (2013). Bilateral basal ganglia activity in verbal working memory. Brain and Language, 125(3), 316-323.

Nee, D. E., \& Brown, J. W. (2013). Dissociable frontal-striatal and frontal-parietal networks involved in updating hierarchical contexts in working memory. Cerebral Cortex, 23(9), $2146-2158$.

Nee, D. E., Brown, J. W., Askren, M. K., Berman, M. G., Demiralp, E., Krawitz, A., \& Jonides, J. (2013). A meta-analysis of executive components of working memory. Cerebral Cortex, 23(2), 264-282.

O’Doherty, J., Dayan, P., Schultz, J., Deichmann, R., Friston, K., \& Dolan, R. J. (2004). Dissociable roles of ventral and dorsal striatum in instrumental conditioning. Science, 304(5669), 452-454.

O’Reilly, R. C., \& Frank, M. J. (2006). Making working memory work: A computational model of learning in the frontal cortex and basal ganglia. Neural Computation, 18(2), 283-328.

Owen, A. M., Iddon, J. L., Hodges, J. R., Summers, B. A., \& Robbins, T. W. (1997). Spatial and non-spatial working memory at different stages of Parkinson's disease. Neuropsychologia, 35(4), 519-532.

Pessoa, L. (2009). How do emotion and motivation direct executive control? Trends in Cognitive Sciences, 13(4), 160-166.

Peters, F., Collette, F., Degueldre, C., Sterpenich, V., Majerus, S., \& Salmon, E. (2009). The neural correlates of verbal short-term memory in Alzheimer's disease: An fMRI study. Brain, 132(7), 1833-1846.

Ranganath, C., DeGutis, J., \& D'Esposito, M. (2004). Category-specific modulation of inferior temporal activity during working memory encoding and maintenance. Brain Research. Cognitive Brain Research, 20(1), 37-45.

Ravizza, S. M., \& Ciranni, M. A. (2002). Set shifting impairments as a function of working memory demands in older adults, prefrontal, and Parkinson's disease patients. Journal of Cognitive Neuroscience, 14(3), 472-483.

Redgrave, P., Rodriguez, M., Smith, Y., Rodriguez-Oroz, M. C., Lehericy, S., Bergman, H., Agid, Y., DeLong, M. R., \& Obeso, J. A. (2010). Goal-directed and habitual control in the basal ganglia: Implications for Parkinson's disease. Nature Reviews Neuroscience, 11(11), 760-772.

Samanez-Larkin, G. R., Worthy, D. A., Mata, R., McClure, S. M., \& Knutson, B. (2014). Adult age differences in frontostriatal representation of prediction error but not reward outcome. Cognitive, Affective, \& Behavioral Neuroscience, 14(2), 672-682.

Serences, J. T., Ester, E. F., Vogel, E. K., \& Awh, E. (2009). Stimulusspecific delay activity in human primary visual cortex. Psychological Science, 20(2), 207-214.

Siegert, R. J., Weatherall, M., Taylor, K. D., \& Abernethy, D. A. (2008). A meta-analysis of performance on simple span and more complex 
working memory tasks in Parkinson's disease. Neuropsychology, 22(4), 450-461.

Swainson, R., Rogers, R. D., Sahakian, B. J., Summers, B. A., Polkey, C. E., \& Robbins, T. W. (2000). Probabilistic learning and reversal deficits in patients with Parkinson's disease or frontal or temporal lobe lesions: Possible adverse effects of dopaminergic medication. Neuropsychologia, 38(5), 596-612.

Sylvester, C.-Y. C., Wager, T. D., Lacey, S. C., Hernandez, L., Nichols, T. E., Smith, E. E., \& Jonides, J. (2003). Switching attention and resolving interference: fMRI measures of executive functions. Neuropsychologia, 41(3), 357-370.

Ventre-Dominey, J., Bourret, S., Mollion, H., Broussolle, E., \& Dominey, P. F. (2014). Dissociable dorsal and ventral frontostriatal working memory circuits: Evidence from subthalamic stimulation in Parkinson's disease. Human Brain Mapping, 35(2), 552-566.

Wilson, F. A., O'Scalaidhe, S. P., \& Goldman-Rakic, P. S. (1993). Dissociation of object and spatial processing domains in primate prefrontal cortex. Science, 260(5116), 1955-1958. 